\title{
Semiconducting phase of amorphous carbon-nickel composite films
}

\author{
Somnath Bhattacharyya, ${ }^{\text {a) }}$ S. J. Henley, D. Lock, N. P. Blanchard, and S. R. P. Silva \\ Nano-Electronics Centre, Advanced Technology Institute, University of Surrey, Guildford GU2 7XH, United \\ Kingdom
}

(Received 16 February 2006; accepted 22 May 2006; published online 12 July 2006)

\begin{abstract}
Amorphous carbon-nickel composite films, which constitute a homogeneously mixed phase of carbon and 10\% nickel, are prepared by UV pulsed laser ablation. From the low temperature conductivity study of these films, a nearly activated conduction followed by conductivity saturation below a temperature of $25 \mathrm{~K}$ has been identified. This is very different from undoped diamond-like carbon (DLC) films. The presence of additional density of states at the Fermi level observed in these samples when compared with DLC films, is directly confirmed using valence band spectroscopy. (C) 2006 American Institute of Physics. [DOI: 10.1063/1.2216030]
\end{abstract}

In the past, many attempts have been made to increase electrical conduction in diamond and amorphous diamondlike carbon (DLC) films by incorporating various light and heavy elements in order to "dope" them and make controllable semiconducting materials. One of the difficulties for substitutional doping of carbon is the formation of unstable graphitic structures, which results in a high defect density of states (DOS) at the Fermi level $\left(E_{F}\right)$. Although several examples of conductivity enhancement of amorphous carbon ( $a$-C) films alloyed with light elements such as nitrogen were found, a true signature of activated or delocalized transport has rarely been demonstrated. ${ }^{1}$ In spite of the unique ability of carbon to mix with a large number of heavy elements, synthesis of a homogeneous amorphous phase of metalcarbon remains difficult. ${ }^{2-5}$ Nickel has been used for the recrystallization of carbon or the graphitization of carbon at high temperature $\left(\sim 500{ }^{\circ} \mathrm{C}\right) .^{2,3}$ In the microstructure of metal and carbon multilayers fabricated by ion bombardment or sputtering, the formation of metal and carbon clusters was attributed to non-homogeneous structures in the films. ${ }^{4}$ Similarly, when mixed phase materials were attempted by substitutional doping of diamond by metal atoms or preparation of diamond-metal composites, they yielded only metallic films having no signature of activated conduction. ${ }^{5}$ Due to the formation of metal clusters and nanostructures, tunneling of electrons was found to dominate the conduction processes even at high temperatures and therefore, an activated conduction, showing a small energy gap at low temperature, was rarely observed. ${ }^{5}$ We show that to achieve a homogeneous amorphous phase or embedded nanostructures, laser ablation can be utilized to mix insulators and metals. Metal catalysts mixed with carbon are routinely used for the growth of various nanostructures such as carbon nanotubes, at high temperature, where supersaturation of the vapor phase of carbon and metal is crucial in the formation of a stable metal-free carbon nanostructure. ${ }^{6}$ It seems that by controlling the temperature and energy, the degree of supersaturation of the carbon-metal mixture can be achieved, giving rise to a metastable amorphous phase. Keeping in mind that the initial state of carbon is important to transform the film structure, we introduce $\mathrm{Ni}$ into highly insulating $s p^{3}$ carbon films by

a) Author to whom correspondence should be addressed; electronic mail: s.bhattacharyya@surrey.ac.uk laser ablation to produce films showing lower resistivity and lower activation energy than the undoped DLC films.

Periodic ablation of a rapidly rotating, high purity, graphite target and nickel foils (both are $99.99 \%$ pure) has been performed using a $248 \mathrm{~nm}$ pulsed UV excimer laser (Lambda Physik LPX 210) delivering a laser fluence of $\sim 10 \mathrm{~J} / \mathrm{cm}^{2}$ at a repetition rate of $10 \mathrm{~Hz}$. The targets were rotated at a constant speed of $\sim 100 \mathrm{rpm}$ and a pressure of $\sim 2 \times 10^{-7}$ Torr was maintained during the deposition at room temperature. The target to substrate distance was fixed at $6 \mathrm{~cm}$. These conditions ensured that during the periodic ablation of each component, the thickness deposited was always less than a monolayer. These samples are approximately $40 \mathrm{~nm}$ thick. From transmission electron microscopy (TEM) performed on a Philips EM400 and field emission scanning electron microscopy (FESEM) the structure of the composite film has been observed. The amount of nickel ( $\sim 10 \%$ ) in the film was estimated from energy dispersive $\mathrm{x}$-ray microanalysis and $\mathrm{x}$-ray photoelectron spectroscopy. $\mathrm{By}$ varying the rotational speed, laser flux, and ratio of $\mathrm{Ni}$ to $\mathrm{C}$ in targets, the percentage of $\mathrm{Ni}$ in the fixed composite films can be controlled to some extent. However, in the present study we focus our efforts on a batch of samples containing $10 \% \mathrm{Ni}$, due to their transport properties. Temperature dependent conductivity has been studied at different temperatures down to $15 \mathrm{~K}$ from samples grown on glass. In addition, $a-\mathrm{C}-\mathrm{Ni} / \mathrm{Si}$ heterostructures were fabricated on samples grown on $n$-type silicon (111) wafers (resistivity $\sim 0.01 \Omega \mathrm{cm}$ ). Ultraviolet photoemission spectra (UPS) (He: excitation energy $=21.22 \mathrm{eV}$ ) of the films, having been annealed (also sputtered) at $\sim 200{ }^{\circ} \mathrm{C}$ to remove adsorbed species from the surface, were obtained at room temperature.

Examining the surface of the as-grown films on silicon wafers we find them to be fairly smooth, uniform, and almost free of delaminated areas. From detailed microscopic studies including TEM and FESEM, no signature associated with crystallites and large clustering of carbon (e.g., graphite) or Ni phases has been observed in the films (Fig. 1). Selected area electron diffraction (SAED) shows only a diffused ring (Fig. 1 inset), which indicates mostly disordered and a homogeneous phase of $a-\mathrm{C}-\mathrm{Ni}$ phase, as opposed to a crystalline or other strongly inhomogeneous structure. In parallel, a detailed analysis of microstructure is under way.

Films prepared without $\mathrm{Ni}$ are diamond-like and highly insulating. Typical conductivity and band gap of the undoped 


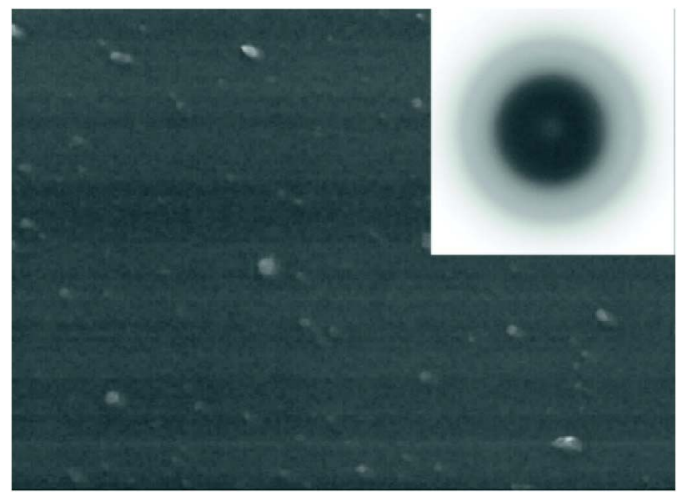

FIG. 1. Typical FESEM micrograph of $a$-C-Ni film. Inset: SAED pattern of the films.

films are $10^{-9} \Omega^{-1} \mathrm{~cm}^{-1}$ and $\sim 2.8 \mathrm{eV}$, respectively, whose temperature dependent conductivity is well researched (for example, see Refs. 1 and 7). On the other hand, the room temperature conductivity of the $a-\mathrm{C}-\mathrm{Ni}$ samples is found to be as high as $10^{2} \Omega^{-1} \mathrm{~cm}^{-1}$ with the estimated value of the band gap much less than that of the undoped DLC films as revealed from the comparison of the optical absorption edges (not shown here). At a first look the variation of conductivity with temperature suggests that an activated electrical process (conductivity changes three orders of magnitude) is dominant over a wide range of temperatures $(50-300 \mathrm{~K})$. From the Arrhenius fit of conductivity an estimation of the activation energy up to $0.05 \mathrm{eV}$ (from $300 \mathrm{~K}$ to $50 \mathrm{~K}$ ) can be made [Fig. 2(a)]. This very low value for DLC films appears to indicate that activated conduction occurs (particularly in the high temperature regime where the conductivity is sufficiently high). In parallel, Mott's variable range hopping (VRH) conduction fits to the data, which is common for DLC films, ${ }^{1,7}$ was found not to fit to the conductivity data of these samples [Fig. 2 inset]. From a crude VRH fit, a very high density of localized states (which do not contribute to the conductivity) may be estimated. However, the large conductivity of the samples and its saturation at low temperature indicate that delocalized conduction within the films may be the most likely explanation. A sharp decrease of the conductivity (up to three orders of magnitude from $300 \mathrm{~K}$ down to $50 \mathrm{~K}$ ) clearly shows that the transport properties of these materials are different from disordered alloys and dilute alloys of magnetic ions $(\mathrm{Ni})$ in a non-magnetic host $(\mathrm{C}){ }^{8}{ }^{8} \mathrm{In}$ -

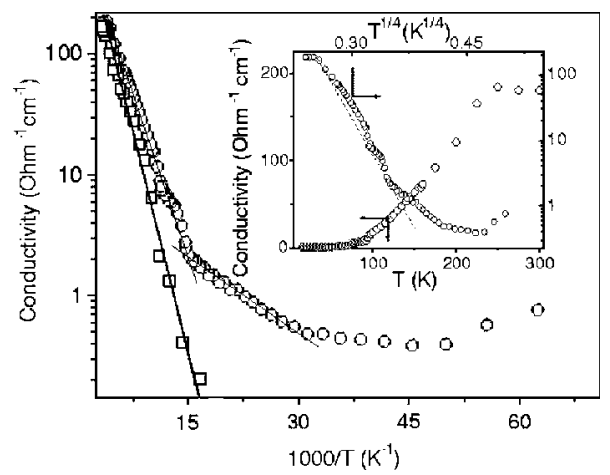

FIG. 2. Low temperature conductivity showing activated behavior of a-C-Ni samples (open squares show activated conduction from another sample having lower concentration of $\mathrm{Ni}$, showing reproducibility of activated conduction). Inset: VRH fit of the data (right and top axis), which does not work well, and conductivity saturation at low temperatures (left and

bottom axis).
Downloaded 30 Mar 2009 to 131.227.178.132. Redistribution subject to AIP license or copyright; see http://apl.aip.org/apl/copyright.jsp

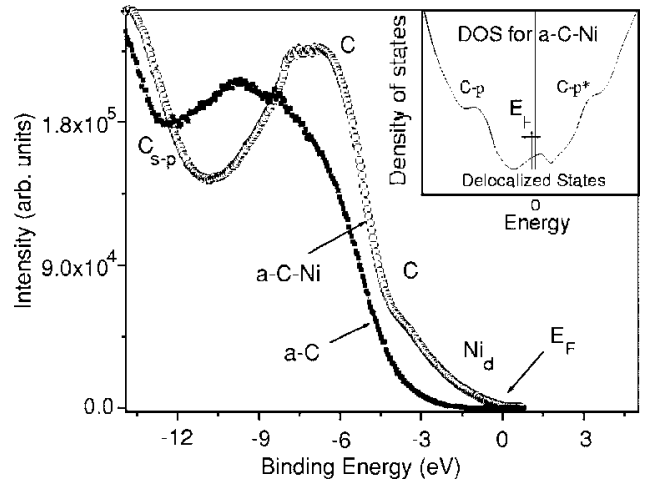

FIG. 3. Ultraviolet photoemission valence band spectra of the $a$-C-Ni films (open circles) and also Ni-free $a-\mathrm{C}$ films (filled squares) as a function of binding energy. Inset: Schematic electronic structure of $a-\mathrm{C}-\mathrm{Ni}$ films proposes finite delocalized states at $E_{F}$.

terestingly, although the amount of Ni is sufficiently high ( $\sim 10 \%$ ), no metal-like temperature dependent conduction in the films can be found, except at very low temperature. The conductivity is relatively low compared with high Ni-rich $a$ -C films (not shown here). The present conductivity trend is opposite to that observed in metal containing diamond, where, due to the presence of granular metal particles, a positive temperature coefficient of resistance was observed over a wide range of temperature. ${ }^{5}$ On the other hand, the VRHtype transport explained by the percolation phenomena, as previously observed in many different forms of metal incorporated carbon films, ${ }^{8}$ is not pronounced in the present carbon-nickel composite films (over the entire range of measurement). Two different slopes in the Arrhenius plot of the conductivity suggest a two-step conduction process due to the change of slope of the DOS and the availability of extra states, which will be discussed. The sharp change of slope is significantly different from a continuous change of slope, which is likely to be seen for VRH conduction at the band tail regions (which is different to the present). The present activated nature of conduction is also different from previous reports on metal incorporated $a$-C films, where a decrease in the resistivity and activation energy with metal percentage, along with a semi-metallic behavior observed at high temperature. $^{7}$ The coefficient of regression calculated for a VRH fit is well below unity (and therefore cannot be considered as a good fit) and also the calculated DOS from the slope of the curve within a limited temperature range gives a large value of defect DOS, which cannot explain the high conductivity of these films and in particular the semimetallic conduction at low temperatures. If the defect DOS is low and edges of bands are delocalized but separated by a small energy barrier it is possible at low temperature to overcome such a barrier as schematically shown in the inset of Fig. 3.

In the low temperature regime, the conductivity deviates from an activated process and a signature of non-zero conductivity is pronounced, as if a borderline metal-insulator transition point is reached. Moreover, the conductivity below $25 \mathrm{~K}$ tends to saturate, followed by a slightly increasing trend when the temperature was further lowered. We note that a similar saturation conductivity at low temperatures has been seen in nanotubes, intercalated graphite fibers, and carbon foils. ${ }^{9-12}$ However, there is a significant difference in the activation energy of the present material. ${ }^{11,12}$ 
When the heterostructure properties of a $a$ $\mathrm{C}-\mathrm{Ni} / \mathrm{Si}$ was measured, $I-V$ characteristics show rectification behavior of up to two orders of magnitude implying semiconducting behavior. A relatively high leakage current may be explained on the basis of a Schottky-type or PooleFrenkel (or hopping) conduction process with Coulomb-like traps. The plots do not show linear behavior, unlike data previously discussed for DLC films. ${ }^{12}$

In order to prepare a homogenous carbon-metal composite film, we examine manners in which to control their microstructure based on the dominance of adatom-substrateatom (adhesive) energy over the adatom-adatom (cohesive) energy. If the cohesive energy exceeds the adhesive energy, small clusters will nucleate on the substrate surface and metal islands will be formed. ${ }^{4-13-15}$ However, when the two energies are equal there is a strong possibility for the formation of homogeneous mixture of the metal and insulator. ${ }^{4}$ Regarding the carbon microstructure, it is well understood that graphitization or the formation of the $s p^{2}$-bonded carbon nanostructures can be enhanced in the presence of metal, where the metal particles act both as a nucleation site and a transport medium for the carbon atoms evaporated by laser ablation. ${ }^{16}$ In order for crystallization to occur, even in nanovolumes, rapid quenching is required to free excess energy, which promotes amorphization, if allowed to release slowly. In the case of a carbon/metal mixture, when cosputtered at low temperature, an amorphous phase is produced. Whereas, crystallization of these materials can occur at high temperature. ${ }^{3}$ We note the temperatures of graphitization by adding $\mathrm{Ni}$ to diamond, and DLC lie between 500 and $900{ }^{\circ} \mathrm{C}$. ${ }^{2}$ In contrast, all our samples were grown at $300 \mathrm{~K}$ to avoid the formation of graphitic or crystalline phase change.

The increase of conductivity in $a$-C films by adding a small quantity of $\mathrm{Ni}$, as previously reported, ${ }^{17}$ can be well understood from the electronic structure of the material using photoelectron spectroscopy. Valence band (VB) photoemission measurements on $\mathrm{Ni}$ films on $a-\mathrm{C}$ or graphite previously showed an intense peak at $\sim 1 \mathrm{eV}$ below $E_{F}$ corresponding to the $3 d^{8}$ band of nickel. ${ }^{18}$ As Ni forms a homogeneous mixture of $\mathrm{C}-\mathrm{Ni}$, instead of forming clusters in these films, no strong peak of $\mathrm{Ni}$ (except a change of slope close to $\sim 0 \mathrm{eV}$ ) is observed in the VB spectra (in Fig. 3) even though the photoionization cross section of the VB electrons for $\mathrm{Ni}$ is more than ten times higher than that of carbon. ${ }^{19}$ On the other hand, features of carbon including $\pi$ and $\sigma$ bands at $\sim 4$ and $\sim 8 \mathrm{eV}$, respectively, are clearly visible from both $a-\mathrm{C}$ and $a-\mathrm{C}-\mathrm{Ni}$ samples ${ }^{20,21}$ (Fig. 3). It is difficult to distinguish the contribution from $\mathrm{Ni}$ or $\mathrm{C}$ in the $\mathrm{VB}$ spectra similar to previous reports. ${ }^{21}$ The most interesting feature of the spectra is the large electron count at $E_{F}$ (here $0 \mathrm{eV}$ ) compared with undoped $a-\mathrm{C}$ films, which clearly demonstrates a finite DOS at $E_{F}$ as proposed from the analysis of low temperature conductivity of the samples (Fig. 2 inset). The electronic structure of the films is depicted schematically (Fig. 3 inset) showing the presence of additional DOS at $E_{F}$. These states may arise due to the formation of $s p^{2}$ bonded carbon by nickel incorporation. Above all, from the conductivity study we think these states could be delocalized and can contribute to the conductivity. In addition, we have studied near edge X-ray absorption fine spectra of $a-\mathrm{C}-\mathrm{Ni}$ films and found a significant difference in the intensity ratio of $\pi^{*}$ to $\sigma^{*}$ peaks compared with DLC films (not shown here).

Demonstration of DLC-based electronic devices has been difficult due to localization. ${ }^{22}$ However, a large decrease of resistivity and activation energy with superior electronic properties, over the undoped DLC films, of a mixed amorphous $\mathrm{C}-\mathrm{Ni}$ phase can be produced by periodic laser ablation of rapidly rotating targets. These results are very different from metal containing diamond or undoped DLC carbon films, where either a semimetallic or variable range hopping conduction process dominates.

The authors would like to thank the EPSRC Portfolio Partnership Award and Carbon Based Electronics Programmes for the financial support.

${ }^{1}$ S. R. P. Silva, J. Robertson, G. A. J. Amaratunga, B. Rafferty, L. M. Brown, J. Schwan, D. F. Franceschini, and G. Mariotto, J. Appl. Phys. 81, 2626 (1997); S. R. P. Silva, B. Rafferty, G. A. J. Amaratunga, J. Schwan, D. F. Franceschini, and L. M. Brown, Diamond Relat. Mater. 5, 401 (1996); Properties of Amorphous Carbon, edited by S. R. P. Silva (INSPEC/IEE, London, 2003).

${ }^{2}$ M. Yadasaka, K. Tasaka, R. Kikuchi, Y. Ohki, and S. Yushimura, J. Appl. Phys. 81, 7623 (1997).

${ }^{3}$ A. G. Ramirez and R. Sinclair, J. Appl. Phys. 85, 5597 (1999); A. G. Ramirez, T. Itoh, and R. Sinclair, ibid. 85, 1508 (1999).

${ }^{4}$ B. L. Evans, A. I. Maaroof, and S. Xu, J. Appl. Phys. 76, 900 (1994).

${ }^{5}$ A. Serra, D. Manno, T. Siciliano, G. Micocci, A. Tepore, M. Rossi, M. L. Terranova, V. Sessa, S. Piccirillo, and S. Orlanducci, J. Appl. Phys. 94, 416 (2003).

${ }^{6}$ M. S. Dresselhaus, G. Dresselhaus, and P. C. Eklund, Science of Fullerenes and Carbon Nanotubes (Academic, New York, 1996); B. O. Boskovic, V. Stolojan, R. U. A. Khan, S. Haq, and S. R. P. Silva, Nat. Mater. 1, 165 (2002).

${ }^{7}$ Rulsi, S. F. Yoon, Q. F. Huang, H. Yang, M. B. Yu, J. Ahn, Q. Zhang, E. J. Teo, T. Osipowicz, and F. Watt, J. Appl. Phys. 88, 3699 (2000).

${ }^{8}$ B. I. Shklovskii and A. L. Efros, Electronic Properties of Doped Semiconductors (Springer, Berlin, 1984).

${ }^{9}$ W. Zhou, J. Vavro, C. Guthy, K. I. Winey, J. E. Fischer, L. M. Ericson, S. Ramesh, R. Saini, V. A. Davis, C. Kittrell, M. Pasquali, R. H. Hauge, and R. E. Smalley, J. Appl. Phys. 95, 649 (2004).

${ }^{10}$ R. T. F. van Schaijk, A. De Visser, S. G. Ionov, V. A. Kulbachinskii, and V. G. Kytin, Phys. Rev. B 57, 8900 (1998); L. Langer, V. Bayot, E. Grivei, J. -P. Issi, J. P. Heremans, C. H. Olk, L. Stockman, C. Van Haesendonck, and Y. Bruynseraede, Phys. Rev. Lett. 76, 479 (1996).

${ }^{11}$ J. Kim, J. O. Lee, H. Oh, K. H. Yoo, and J. J. Kim, Phys. Rev. B 64, 161404 (2001).

${ }^{12}$ A. Bozhko, A. Ivanov, M. Berrettoni, S. Chudinov, S. Stizza, V. Dorfman, and B. Pypkin, Diamond Relat. Mater. 4, 488 (1995).

${ }^{13}$ C. Ronning, U. Greismeier, M. Gross, and H. C. Hofsass, Diamond Relat. Mater. 4, 666 (1995).

${ }^{14}$ I. Gerhards, C. Ronning, H. Hofsass, M. Seibt, and H. Gibhardt, J. Appl. Phys. 93, 1203 (2003).

${ }^{15}$ J. Jiao, S. Seraphin, X. Wang, and J. C. Withers, J. Appl. Phys. 80, 103 (1996).

${ }^{16}$ J. P. Zhao, Z. Y. Chen, X. Wang, and T. S. Shi, J. Appl. Phys. 87, 8098 (2000).

${ }^{17}$ J. C. Orlianges, C. Champeaux, A. Catherinot, A. Pothier, P. Blondy, P. Abelard, and B. Angleraud, Thin Solid Films 453, 291 (2004).

${ }^{18}$ N. Martensson and B. Johansson, Phys. Rev. Lett. 45, 482 (1980).

${ }^{19}$ E. Barborini, C. Lenardi, P. Piseri, P. Milani, R. G. Agostino, T. Caruso, E. Colavita, S. La Rosa, M. Bertolo, and C. Ducati, Eur. Phys. J. D 24, 273 (2004).

${ }^{20}$ J. Schafer, J. Ristein, R. Graupner, L. Ley, U. Stephan, Th. Frauhenheim, V. S. Veeraswamy, G. A. J. Amratunga, M. Weiler, and H. Ehrhardt, Phys. Rev. B 53, 7762 (1996).

${ }^{21}$ S. Bhattacharyya, C. Cardinaud, and G. Turban, J. Appl. Phys. 83, 4491 (1998); S. Bhattacharyya, C. Spaeth, and F. Richter, ibid. 89, 2414 (2001).

${ }^{22}$ S. Bhattacharyya, S. J. Henley, E. Mendoza, L. G. Rojas, J. Allam, and S. R. P. Silva, Nat. Mater. 5, 19 (2006). 